\title{
28. RADIO OBSERVATIONS OF SOME DETAILS IN THE HI LOCAL SPIRAL ARM
}

\author{
N. V. BYSTROVA, J. V. GOSSACHINSKY, T. M. EGOROVA, \\ V. M. ROZANOV*, and N. F. RYZHKOV \\ Pulkovo Observatory, Leningrad, U.S.S.R.
}

The observations were made with the large Pulkovo radiotelescope (beamwidth $7^{\prime}$, bandwidth 20 and $10 \mathrm{kHz}$ ). Figure 1 contains our drift curves across the cluster NGC 2264. The details on them may be identified with Raimond's (1966) clouds, but the proofs for the reality of the connection between the cluster and the clouds are not very reliable. The cloud ' $b$ ' is believed to be connected with NGC 2264 because of their close neighborhood. But on the other side of the galactic plane in this region we have found a bright and narrow $\left(\sim 1^{\circ}\right)$ detail, whose middle falls exactly on the

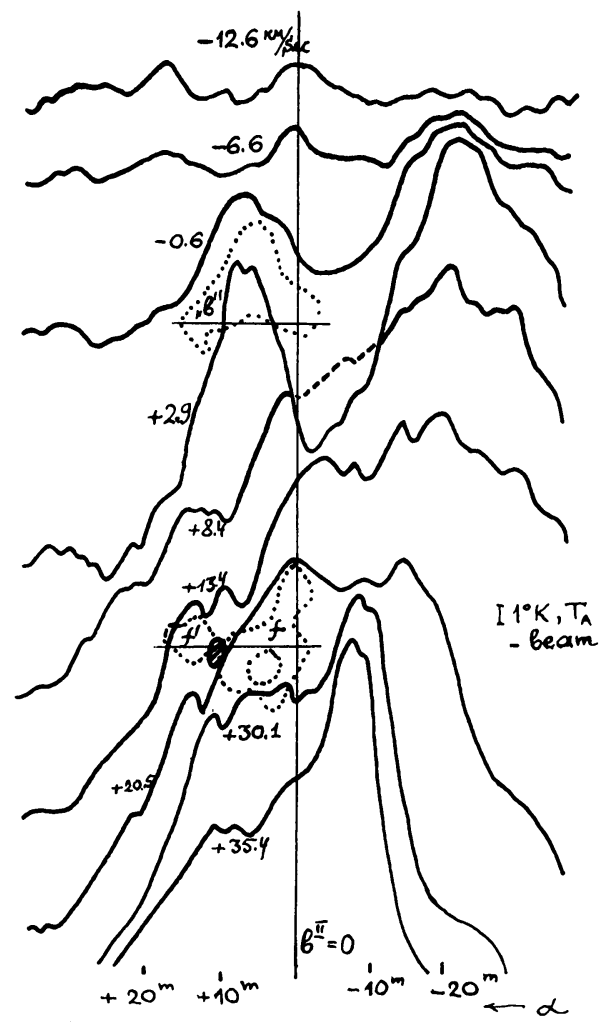

Fig. 1. Drift curves across the cluster NGC 2264 at different velocities relative to the LSR. The coordinates are RA and antenna temperature. Dots represent the contours of the clouds found by Raimond, a line across them represents our scans.

* V. M. Rozanov died on August 12, 1968, at the age of 29. 
western border of the $\mathrm{H}$ II region around the star $\lambda$ Orionis (Figure 2). Figure 3 contains the computed drift curves at different velocities for $\Delta f=20 \mathrm{kHz}$ according to the model of the neutral hydrogen expanding envelope proposed by Wade (1957) around the HII region $\lambda$ Ori. Figure 4 represents our observations. Although some details, in particular the narrow detail at the velocity $-1.4 \mathrm{~km} \mathrm{~s}^{-1}$, are very similar to the calculated ones, our observations differ from Wade's model in that they do not have

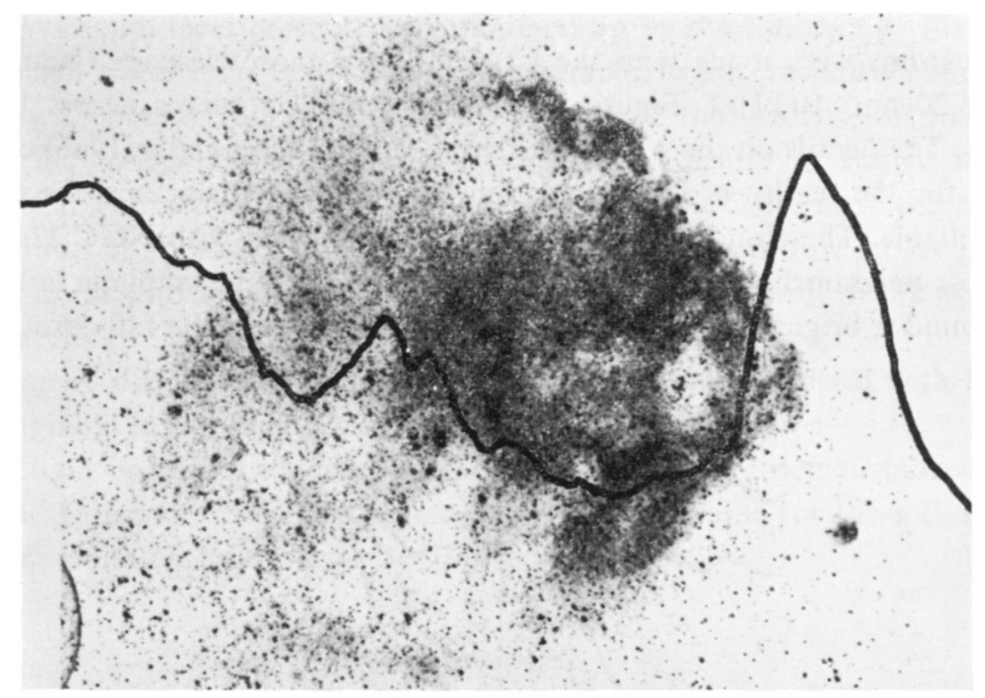

Fig. 2. Part of the drift curve at velocity $-1.4 \mathrm{~km} \mathrm{~s}^{-1}$ across the star $\lambda$ Ori, put on the photograph of this $\mathrm{HII}$ region.

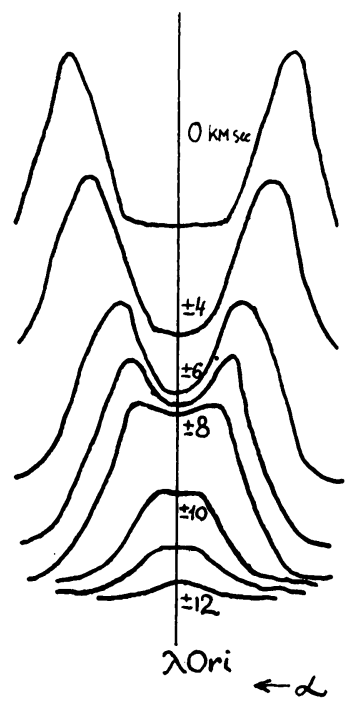

Fig. 3. Expected drift curves across $\lambda$ Ori according to the model by Wade (1957) for a $20 \mathrm{kHz}$ band. 
any velocity symmetry relative to zero; in fact the details are situated only at the positive side. This fact may eliminate one half of the proposed envelope. There is no symmetry relative to the central star and no coincidence of the velocity of the narrowest detail with the velocities of the central star and nebula. Figure 5 demonstrates the drift curve obtained across both objects, NGC 2264 and $\lambda$ Ori nebula. Maybe the old Menon's idea about the connection between the branching of the gaseous arm and the formation of the clusters is correct. The details in the Hi distribution look like the split branches from the main body of the arm (Figure 5).

On Figure 6 are our drift curves across the region of the magnetic field reversal near $l^{\mathrm{II}}=180^{\circ}, b^{\mathrm{II}}=-30^{\circ}$ (Gardner et al., 1967). Taking into account the model on Figure 2 we may conclude that there is a hole with diameter approximately $50 \mathrm{pc}$ in

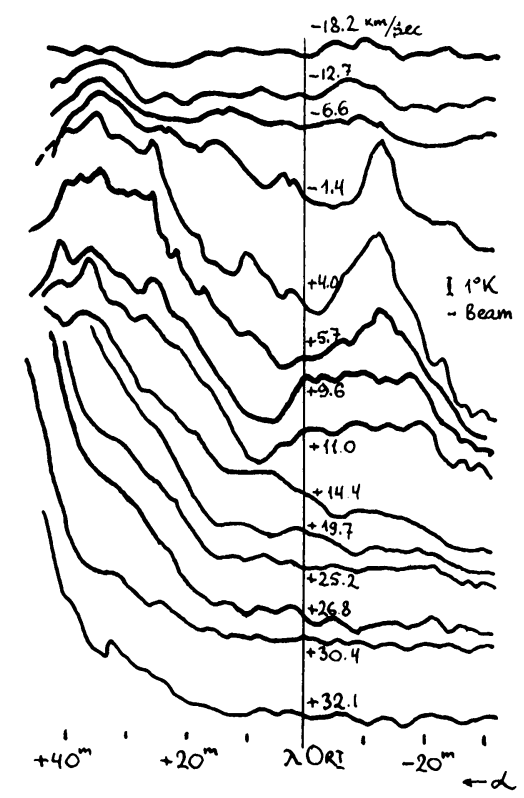

Fig. 4. Observed drift curves at different velocities across $\lambda$ Ori.

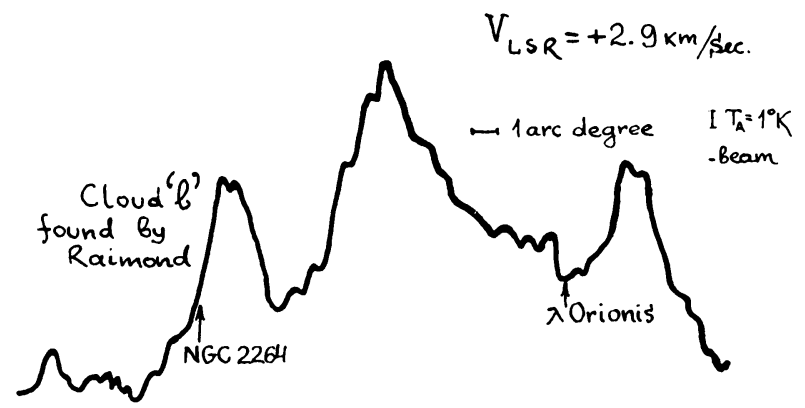

Fig. 5. Drift curve across NGC 2264 and across the HII region $\lambda$ Orionis. $V=+2.9 \mathrm{~km} \mathrm{~s} \mathrm{~s}^{-1}$ relative to $\mathrm{LSR}, \Delta f=10 \mathrm{kHz}$. 


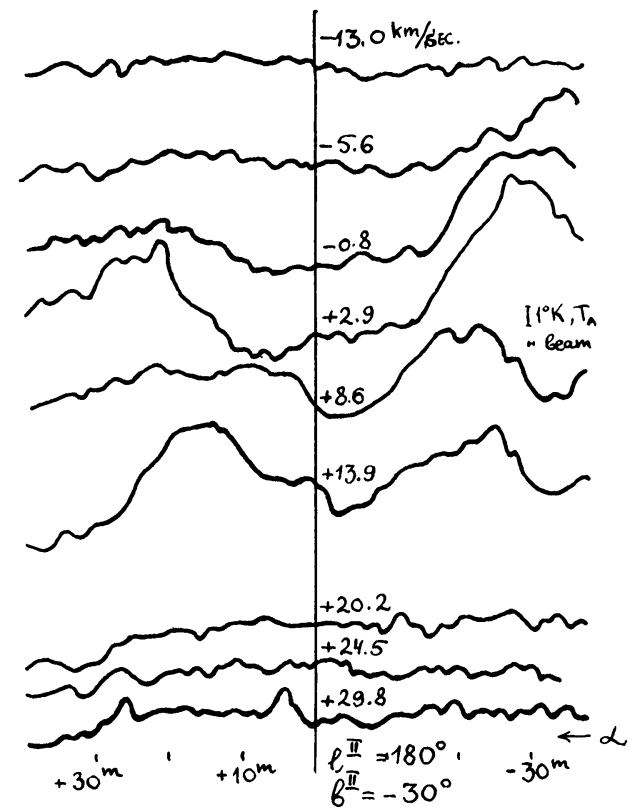

Fig. 6. Drift curves across the region of magnetic field reversal at $l=180^{\circ}, b=-30^{\circ}$.

the gaseous tongue in question. Here may be something like a half-envelope expanding with the velocity $12-13 \mathrm{~km} \mathrm{~s}^{-1}$.

\section{Acknowledgements}

Our thanks are due to Z. A. Alferova for the programming of observations and for further computer reductions and to N. S. Evgrafov and V. A. Jakovlev for their taking part in the observations.

\section{References}

Gardner, F. F., Whiteoak, J. B., and Morris, D.: 1967, Nature 214, 371.

Raimond, E.: 1966, Bull. Astron. Inst. Netherl. 18, 191.

Wade, C. M.: 1957, Astron. J. 62, 148. 